\title{
Theories of Knowledge
}

\section{Evidence-based practice: reflections from five European case studies}

\author{
Juan I Baeza and Alec Fraser \\ Department of Management, King's College London, UK \\ Annette Boaz \\ Centre for Health and Social Care Research, St Georges, University of London, UK
}

\section{Key message(s)}

- Evidence-based practice (EBP) needs to draw on a wide range of evidence that is not only based on randomized controlled trials.

- The location of practice (acute, community or primary care) needs to be carefully considered when using EBP.

- Inter- and intraprofessional relations need to be carefully considered in relation to EBP.

\section{Why this matters to me}

Through our European study, we have learnt that the nature of evidence is a contested term that can both bring different professionals together, for example, within stroke units, or harm professional relationships, for example, between stroke units and primary and community care. For evidence-based practice to have a positive impact on patients, it needs to be used as an inclusive endeavour rather than an exclusive one.

\section{Related LJPC papers}

Toon and Thomas (both in this issue).

\section{ABSTRACT}

Background Evidence-based practice (EBP) is now the accepted orthodoxy in clinical practice and developed from evidence-based medicine. EBP is based on a specific type of evidence that is derived from studies based on randomised controlled trials (RCT). This type of evidence is suited to acute medical care and is more problematic for other clinicians such as nurses and therapists, particularly when they are situated within community or primary care settings.

Setting Five stroke care services in England (2), Sweden (2) and Poland (1).

Aims To reflect on the evidence gained from these case studies to shed light on various aspects of EBP. This paper focuses on three key issues: (1) the importance of context for evidence, (2) the nature of knowledge, and (3) professional hierarchies.

Methods Five qualitative case studies into stroke care were carried out in England, Sweden and Poland. One hundred and twenty semi-structured interviews were carried out with a range of healthcare staff who provided specialised and non- specialised stroke care in acute, community and primary care between October 2010 and September 2011. Medical doctors, nurses and different therapists were included in the samples in all five case studies. For this paper, we reflect on some aspects of this work to illuminate the different interprofessional perspectives relating to EBP in stroke care.

Results The lack of RCT-based evidence in the community and primary care sectors can lead to the clinicians working in these sectors being perceived as having a lower status. Clinicians use both tacit and encoded knowledge to guide their practice and there existed both intra- and interprofessional tensions in these two types of knowledge. The professional hierarchy of stroke teams varies with national context and the role of the nonspecialists is less valued in stroke care.

Keywords: Europe, evidence-based medicine, evidence-based practice, stroke care 


\section{Introduction}

Evidence-based medicine (EBM) was initially a movement that centred upon the medical profession and medical practice - it is a 'medical model' of evidence that considers 'encoded' facts, one at a time, in isolation from their context. As Dopson puts it:

EBM changes medical practice from being primarily based on the accumulation of clinical experience, observation and expertise to a model that can be characterised as a systematic and rigorous examination of relevant scientific evidence. ${ }^{1}$

Then, as Timmermans points out, ${ }^{2}$ EBM spread to other healthcare professionals to become evidencebased practice (EBP), which is more inclusive of the work that all healthcare professionals are engaged in. EBP has become the accepted orthodoxy in clinical practice within healthcare systems the world over. ${ }^{3}$ In general, EBP has widespread support among clinicians. ${ }^{1}$

One of the key attributes of EBP is that not all evidence is considered equivalent; there is a clear hierarchy of evidence that is dependent on the research design and its implied 'validity', which is itself a contested term. ${ }^{4}$ The randomised controlled trial (RCT) sits at the top of this hierarchy; nonrandomised controlled trials, case studies and observational studies occupy lower ranks on the EBP ladder, while qualitative studies are almost disregarded in terms of evidence and their status is considered comparable with ideas and opinions. ${ }^{5}$ As Toon discusses in another paper on evidence in this issue, ${ }^{6}$ rather than relying on single studies, EBP prefers to draw on a wide range of evidence gathered from a number of rigorously conducted research studies on a particular topic; systematic reviews are carried out to produce this. The Cochrane Collaboration that was established in 1993 generates these systematic reviews on a large range of topics (www.cochrane.org). The pioneers of EBM defend this hierarchy of evidence:

Because the randomised trial, and especially the systematic review of several randomised trials, is so much more likely to inform us and so much less likely to mislead us, it has become the 'gold standard' for judging whether a treatment does more harm than good. (p. 71) ${ }^{7}$

Although there have been various criticisms of the $\mathrm{RCT},{ }^{4,8,9}$ this method remains at the pinnacle of the evidence hierarchy, giving an automatic bias to healthcare provision that is amenable to the RCT. This bias can work against many therapy services in general and community-based therapy services in particular. For example, a study into evidence and the provision of physical therapies for young children with motor disabilities reported that of 444 intervention study papers, only 31 met the criteria for evidence. ${ }^{10}$ The lack of good quality RCTs for interventions carried out by therapists is often cited in the literature, ${ }^{10,11}$ while others criticise some EBP for being inappropriate within actual clinical practice, arguing that there is a fundamental clash between the 'research paradigms' and the 'therapy paradigms' ${ }^{12}$ They argue, as does Bayliss (described in a partner paper in this issue of $L J P C$ ), that the medical model of evidence does not have the power to recognise the complexities involved in much of the work of therapies. $^{13,14}$

It is well known that clinical practice cannot be described solely by encoded evidence. Greenhalgh et $a^{15}$ argue that clinical action is the result of the synthesis between professional judgement (tacit knowledge) and formal rule-based systems such as EBP (encoded knowledge); they concluded that encoded knowledge on its own was not a sufficient base for clinical action. However, the tensions and balance between the tacit and encoded knowledge may be different for the different professional groups. Continuing on this theme, Timmermans suggests that the EBM movement can be seen as a shift from disciplinary to mechanical objectivity, where disciplinary objectivity is associated with tacit knowledge and mechanical objectivity is associated with rules and procedures or encoded knowledge that make up EBP. ${ }^{2}$

In terms of power, it has been argued that the medical profession has replaced the 'disciplinary power' it claimed before EBM (by virtue of having high levels of tacit knowledge through being more educated and experienced than others) with 'mechanical power' (more skilled at the use of EBP than others), and this has strengthened doctors' professional dominance over other professional groups within health systems. ${ }^{16}$ As Armstrong et al $l^{17}$ argue, "What is counted, how it is counted, how it is processed, and what is done with what is found are value and power-laden choices' (p. 132).

Following the details of the methods employed, this paper discusses the limitations of EBP outlined above by reflecting on the evidence gained from five case studies of stroke care in three European countries, namely: England, Sweden and Poland.

\section{Methods}

Five comparative case studies of the implementation of evidence-based stroke care in England, Sweden and Poland were conducted. The case studies focused on stroke units but included community and general practice (GP) services in England (two hospital sites), Sweden (two hospital sites) and Poland (one hospital site), which comprised 120 interviews, with both internal and external validity. ${ }^{18,19}$ 
England, Sweden and Poland were selected following discussions with European stroke specialists who make up the European Implementation Score Collaborative Group. According to national audit data, Sweden and England represent different levels of development in the delivery of stroke services; Sweden is thought to have one of the most highly developed services, whereas stroke services in England are in a more developmental stage following the implementation of a national stroke strategy in 2007. Poland is the least developed in terms of its stroke services.

Table 1 shows the spread of interviews conducted in each of the case studies. Fuller accounts of these case studies can be found in another paper by the authors. $^{20}$

These five case studies allowed us to investigate a number of important factors related to translating research evidence into healthcare practice in the area of stroke care. Through interviews with medical doctors, nurses and therapists, the original research examined what organisational aspects might help or hinder an organisation's ability to successfully transfer research evidence into practice in stroke services, ${ }^{21,22}$

In this paper, we reflect on aspects of this work to consider what we learned about the differential interprofessional perspectives to EBP in stroke care. We examined three issues related to EBP:

- the importance of context for evidence;

- the nature of knowledge; and

- professional hierarchies.

These areas are addressed briefly below; a more substantive paper on these issues is under preparation by the research team.

\section{The importance of context for evidence}

From the case studies, it was clear that the type and location of evidence were interconnected. The lack of RCTs and their limitations outside the acute stroke sector (particularly in primary and community care) illustrate how the type of evidence (RCT) and the location (acute versus non-acute care) are linked. A consequence of the lack of RCT-derived evidence in the non-acute sector is that the evidence base in primary or community care is perceived as low in EBP terms. Therapists, in particular, often argued that it was difficult to find the required level of evidence that directly related to their day-to-day practice. This perceived lack of evidence for therapists' work was viewed as being particularly problematic by those in the community sector, illustrating the importance of location of practice. The importance of context in
Table 1 Case study interviews

\begin{tabular}{ll}
\hline Case study site & $\begin{array}{l}\text { Number of } \\
\text { interviews }\end{array}$ \\
\hline England case study 1 (urban) & 25 \\
England case study 2 (rural) & 20 \\
Sweden case study 2 (rural) & 28 \\
Sweden case study 1 (urban) & 22 \\
Poland case study 1 (urban) & 25
\end{tabular}

terms of evidence is thoughtfully considered in Thomas' paper, which is also in this issue. ${ }^{14}$

It would seem that one possible solution to the evidence deficit in therapists' work would be for them to directly engage in research. However, this was seen as being too difficult to incorporate into their current roles. By contrast, research was seen as very much part of a hospital doctor's job. These perceptions may explain why 'doctor-derived' evidence was thought to be privileged over other types of evidence.

There was also evidence in the case studies of intraprofessional tensions within the medical profession on the perceived strength of evidence for certain practices; differences of opinion, for example, on what was and what was not EBP were reported between radiologists and stroke physicians. Another intraprofessional tension that was detected centred on the location of practice, between the hospital setting of the stroke consultant and the primary care setting of the family doctor. Family doctors felt that a lot of the EBM derived from the hospital could not always be applied to their general practice population.

The insights gained from the case studies suggest that RCT, acute, medical type evidence is what is valued in stroke care. The lack of this type of evidence in the community and primary care sectors may lead to the clinicians working in these sectors being perceived as having a lower status within the dominant EBP context. The type and location of evidence are also closely linked to the twin concepts of tacit and encoded knowledge, as discussed below.

\section{The nature of knowledge}

The case studies showed that therapists tended to work at the tacit range of the knowledge spectrum. They explained their 'non-evidenced-based practice' by stating that they dealt with how patients actually functioned in front of them and not on what the 'scans 
say'. Some therapists and general nurses explained (and defended) this tacit-based knowledge approach as one that was guided by 'what works'. Again, this tension between the use of tacit and encoded knowledge was not only confined to the therapists, it seemed that GPs and A\&E consultants also shared this tension about the nature of knowledge. The case studies showed that all the professions used a mixture of tacit and encoded knowledge in their practice, but the therapists tend to rely more on tacit knowledge. They also showed that the use of encoded knowledge tends to be inversely related to the age of clinicians. As Thomas also states in this issue, ${ }^{14}$ multi-professional teams deliver much of modern healthcare so it is important to consider the internal dynamics of these teams in terms of their views of knowledge.

\section{Professional hierarchies}

Although many professionals reported good interprofessional relations and working practices, the Polish case study illustrated clear divisions between doctors and other professions, displaying a clear professional hierarchy between doctors and nurses. For example, stroke nurse specialists do not exist in Poland; with Polish nurses having a very low status compared with doctors and often being side-lined in decision-making and research. However, there were some signs that this professional inequality may be slowly changing as nurses gain higher and better qualifications in Poland.

It would be wrong to conclude that this medical dominance is only evident in Poland, it also seems to exist, albeit to a lesser degree, in England where various illustrations of negative views of the abilities of the general nursing profession were reported in the English case studies. These negative perceptions of some nurses and their marginal role in terms of research could perhaps be structural, as they reported difficulties in taking part in research meetings due to a pivotal role on the ward that also makes it difficult for them to take part in training. However, this seems to be a contextual feature that was not present for the nurses in the Swedish case studies. The hierarchy of stroke teams seems to vary with national context but it would seem that the role of the non-specialist, be they medical doctors, nurses or therapists, is not valued in this specialised area of healthcare.

\section{Discussion}

Although EBP is an important aspect of modern healthcare, it needs to reflect and be made relevant to the clinical work of therapists, which often entails multiple rather than single interventions that may not have been subject to a RCT. ${ }^{12}$ Many desired outcomes are simply not addressed in the evidence literature because many of the therapists' interventions do not aim to cure or even alleviate symptoms and so fall outside the 'medical model' that is the central foundation of EBP, making the RCT gold standard inappropriate for many of the therapists' interventions. ${ }^{10,16}$ This observation concurs with that of the Bayliss et al ${ }^{13}$ that dynamic multifaceted phenomena (such as stroke care) require research approaches that are 'participatory, mixed methods, multi-level, and engage communities'.

EBM developed from a decline in trust that could be seen as being society-wide and from a demand for greater accountability, ${ }^{3}$ and as Timmermans argues, ${ }^{2}$ EBM now serves a number of other less progressive purposes by providing:

... a dominant and sweeping social mechanism to control unruly individual professionals, regain the public's trust, and shore up the scientific quality of the professional medical project that has spread from physicians to other allied health professions. (p. 167)

In addition, as Harrison argues, EBM has an important political feature:

EBM is not a purely scientific endeavour. It has an important political dimension, what EBM is and how it is defined is contested and hence political. ${ }^{5}$

However, in spite of these threats, the status of specialist therapists from Poland and England does not seem to have been damaged by their non-evidence based ways of working. It seems that specialisation (and not professional background) is the important determinant of status in stroke care. It would appear that general nurses are the ones who are most effected by EBP in terms of their status, particularly in very hierarchical health systems such as that found in Poland. Although, as was seen in Sweden, this is not the case for specialist stroke nurses, so there are important intraprofessional differences in relation to EBP.

Drawing on the reflections from this stroke study, it is clear that a number of inter- and intraprofessional tensions exist with EBP that are related to geographical and clinical context. However, these tensions are dynamic and subject to change.

\section{GOVERNANCE}

The research that this paper is based upon gained all the relevant ethical approvals in the countries where the case studies were conducted. 


\section{CONFLICT OF INTEREST}

None declared.

\section{ACKNOWLEDGEMENTS}

The authors would like to express their thanks Dr Paul Thomas for providing very helpful guidance on the development of this paper.

\section{REFERENCES}

1 Dopson S, Locock L, Gabbay J, Ferlie E and Fitzgerald L (2003) Evidence-based medicine and the implementation gap. Health 7:311-30.

2 Timmermans S (2008) Professions and their work: do market shelters protect professional interests? Work and Occupations 35:164-88.

3 Lambert H (2006) Accounting for EBM: notions of evidence in medicine. Social Science \& Medicine 62: 2633-45.

4 Grossman J and Mackenzie F (2005) The randomized controlled trial: gold standard, or merely standard? Perspectives in Biology and Medicine 48:516-34.

5 Harrison S and McDonald R (2008) The politics of healthcare in Britain. Sage: London.

6 Toon P (2014) What is evidence? London Journal of Primary Care 6:95-7.

7 Sackett D, Rosenberg W, Gray J, Haynes R and Richardson W (1996) Evidence-based medicine: what it is and what it isn't. British Medical Journal 312:71-2.

8 Borgerson K (2005) Evidence-based alternative medicine? Perspectives in Biology and Medicine 48:502-15.

9 Bluhm R (2005) From hierarchy to network: a richer view of evidence for evidence-based medicine. Perspectives in Biology and Medicine 48:516-34.

10 Landsman G (2006) What evidence, whose evidence?: Physical therapy in New York State's clinical practice guidelines and the lives of mothers of disabled children. Social Science \& Medicine 62:2670-80.

11 Leung E (2002) Evidence-based practice in occupational therapy. Hong Kong Journal of Occupational Therapy 12:21-32.

12 Grimmer K, Bialocerkowski A, Kumar S and Milanese S (2004) Implementing evidence in clinical practice: the therapies' dilemma. Physiotherapy 90:189-94.

13 Bayliss $\mathrm{E}$ et al (2014) Understanding the context of health for persons with multiple chronic conditions: moving from what is the matter to what matters. Annals of Family Medicine 12: 260-9. http://www.annfammed. org/content/12/3/260.full

14 Thomas P (2014) Understanding context in healthcare research and development. London Journal of Primary Care 6:103-5.

15 Greenhalgh J, Flynn R, Long A and Tyson S (2008) Tacit and encoded knowledge in use of standardised outcome measures in multidisciplinary team decision making: a case study of in-patient neurorehabilitation. Social Science \& Medicine 67:183-94.

16 Light DW (1995) Countervailing powers: a framework for professions in transition. In: Johnson T, Larkin G and Saks M (eds) Health professions and the State in Europe. Routledge: London, pp. 25-41.

17 Armstrong P, Armstrong H and Coburn D (2001) Unhealthy times. Political perspectives on health and care in Canada. Oxford University Press: Oxford.

18 Eisenhardt K (1989) Building theories from case study research. Academy of Management Review 14:532-50.

19 Langley A (1999) Strategies for theorising from process data. Academy of Management Review 24:691-710.

20 Baeza J, Boaz A, Fraser A, Fulop N, McKevitt C and Wolfe C (2012) The importance of normative integration in stroke services: case study evidence from Sweden and England. Health Services Management Research 25:155-61.

21 Baeza J, Boaz A and Fraser A (2012) The European Implementation Score (EIS) - a novel tool for identifying factors successful in research implementation into clinical practice. Invited presentation at the XXI European Stroke Conference, 22-25 May, Lisbon.

22 Baeza J, Boaz A and Fraser A (2012) The role of case studies in investigating the gap between research, policy and practice. Paper presented at the 16th Annual Conference of the International Research Society for Public Management, 11-13 April, Rome.

\section{ADDRESS FOR CORRESPONDENCE}

Dr Juan Baeza

Department of Management

King's College London

150 Stamford Street

London SE1 9NH

Tel: 02078484634

Email: juan.baeza@kcl.ac.uk

Received June 2014, revised July 2014, accepted August 2014 